\title{
Traveling waves in a spring-block chain sliding down a slope
}

\author{
J. E. Morales, G. James and A. Tonnelier \\ INRIA-Grenoble 655 avenue de l'Europe, Montbonnot \\ 38334 Saint Ismier, France *
}

(Dated: 27 July 2017)

\begin{abstract}
Travelling waves are studied in a spring slider-block model. We explicitly construct front waves (kinks) for a piecewise-linear spinodal friction force. Pulse waves are obtained as the matching of two traveling fronts with identical speeds. Explicit formulas are obtained for the wavespeed and the wave form in the anti-continuum limit. The link with localized waves in a Burridge-Knopoff model of an earthquake fault is briefly discussed.
\end{abstract}

\section{INTRODUCTION}

Spatially discrete systems (lattice differential equations) have a wide range of applications in natural sciences, engineering and social sciences. They frequently occur in physics as mass-spring systems with nearestneighbors coupling and they have been used extensively to describe the dynamics of microscopic structures such as crystals or micromechanical systems [1-3], or to model fragmentation phenomena [4]. Recent studies on soft structures have led to a renewed interest in the dynamics of elastically coupled systems with a special emphasis on transition waves [5].

In this work, we consider a spring-block system that slides down a slope due to gravity (see Fig. 1). Each block is subjected to a nonlinear friction force. This system differs from the Burridge-Knopoff model [6] considered for the modeling of earthquakes, which incorporates local potentials.

We consider here a friction force of spinodal type: the steady-state kinetic friction coefficient has a nonmonotonic profile versus sliding velocity such as the one depicted in Fig.2. Such friction laws have been reported to induce excitable dynamics (see [7] and references therein) reminiscent of neural excitability [8, 9], i.e., a perturbation above a certain threshold produces a large excursion in the phase space before returning to an equilibrium state. In biology, it is well documented that a large class of excitable media is able to support nonlinear solitary waves [10]. It has been recently shown that excitable mechanical systems also have the ability to induce self-sustained solitary waves [11-13]. In contrast with classical excitable media, these systems are elastic rather than diffusive.

The analysis of traveling patterns in discrete media often relies on continuum approximations. In the slider block model presented here, we directly tackle the discrete nature of the equations and use an idealized piecewiselinear friction force to derive semi-analytical expressions for propagating waves. This "bilinearization" approach has been used in a variety of contexts to study traveling waves in lattices, see e.g. [14-23].

*guillaume.james@inria.fr, arnaud.tonnelier@inria.fr
The paper is organized as follows. In Sec II, we first derive the governing equations for the chain of elastically coupled blocks. Then in Sec. III we study the dynamical properties of an isolated block and demonstrate that a bistable behavior exists when a spinodal friction force is considered. In Sec. IV, we perform numerical simulations of the coupled system and show that the bistability property induces traveling patterns, as fronts and pulses. In Sec. V, we construct the traveling fronts analytically using a piecewise-linear friction force. The anticontinuum limit is presented in Sec. VI. The link between front and pulse waves is studied in Sec. VII. We then conclude by connecting the results to the Burridge-Knopoff model.

\section{MODEL}

Let us consider an isolated block of mass $m$ and position $x(t)$ that slips down a slope under gravity and is subject to a velocity-dependent friction force $F\left(\frac{d x}{d t}\right)$. The dynamical equations read

$$
m \frac{d^{2} x}{d t^{2}}+F\left(\frac{d x}{d t}\right)=G
$$

where $G$ is the tangential component of the gravity force. A steady state of (1) exists when the block achieves a constant velocity motion $\frac{d x}{d t}=V$, where $F(V)=G$. Let us consider an infinite chain of identical blocks linearly coupled through Hookean springs of stiffness $k$ that slips at the constant speed $V$ over an inclined surface (see Fig. 1). The dynamical equations in a frame moving at velocity $V$ are given by

$$
\begin{aligned}
\frac{d y_{n}}{d t} & =u_{n}, \\
m \frac{d u_{n}}{d t} & =k \Delta_{d} y_{n}-F\left(V+u_{n}\right)+G, \quad n \in \mathbb{Z}
\end{aligned}
$$

where $y_{n}$ represents the displacement of the $n$th block from the steady sliding state, and $u_{n}$ is its velocity. The term $\Delta_{d} y_{n}=y_{n+1}-2 y_{n}+y_{n-1}$ is the discrete Laplacian.

The system may be interpreted as a variant of the Burridge-Knopoff model [6] where the shear stress described by the local potential is replaced by a constant 


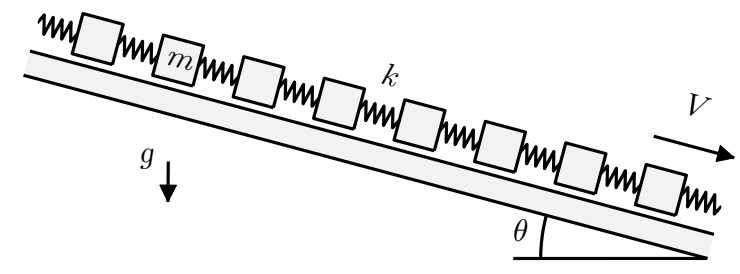

FIG. 1. Mechanical representation of the block-spring slider model where $m$ is the mass, $k$ is the spring constant and $V$ is the sliding velocity. The steady state corresponds to $F(V)=G$ with $G=m g \sin \theta$.

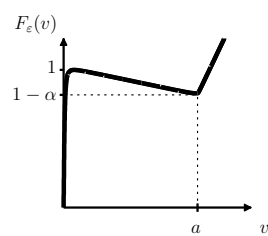

(a)

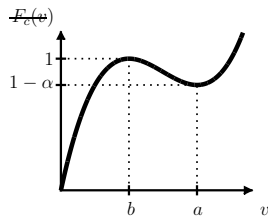

(b)

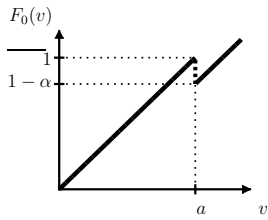

(c)
FIG. 2. Non-monotonic friction laws. (a) Coulomb-like friction force $F_{\varepsilon}$, where $\varepsilon=10^{-4}$. (b) The cubic friction force $F_{c}(v)$, where $b=0.5, a=1$ and $\alpha=0.2$. (c) The piecewise linear friction force $F_{0}(v)$.

tangential force induced by gravity. The dynamics of system (2) is explored for three normalized non-monotonic friction laws $F_{\varepsilon}, F_{c}$ and $F_{0}$, depicted in Fig. 2(a-c) and given by

$$
\begin{aligned}
& F_{\varepsilon}(v)=[1-\alpha+\sqrt{N(v)}] \frac{v}{\sqrt{\varepsilon+v^{2}}}, \\
& F_{c}(v)=3.2 v^{3}-7.2 v^{2}+4.8 v \\
& F_{0}(v)=v / a-\alpha H(v-a)
\end{aligned}
$$

where $N(v)=\varepsilon+4 \max (|v|-a, 0)^{2}+\alpha^{2} \max (a-|v|, 0)^{2}$, and $H$ is the Heaviside step function. For convenience, the cubic friction force $F_{c}$ is given for $a=1$ where $a$ is the location of the local minimum, i.e. the transition point from the velocity-weakening $(b<v<a)$ to the velocity-strenghtening $(v>a)$ regime. The friction function $F_{\varepsilon}$ describes a regularized generalized Coulomb law as $\varepsilon \rightarrow 0$. The cubic friction force $F_{c}$ describes a smooth spinodal friction law similar to the one introduced in [11]. The piecewise linear function $F_{0}$ reduces the velocity-weakening region to a jump discontinuity. It captures some properties of spinodal friction laws and is convenient for analytical computations.

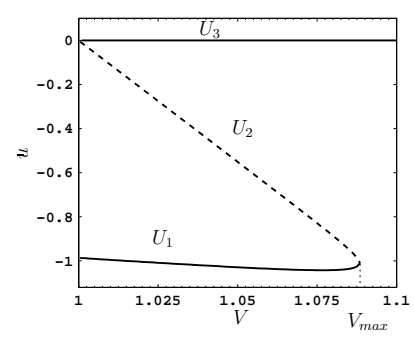

(a)

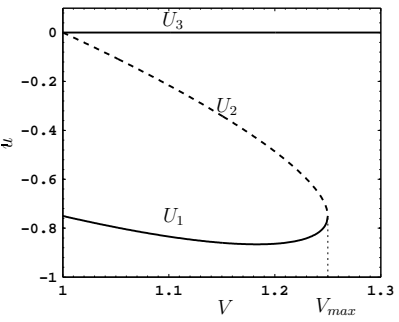

(b)

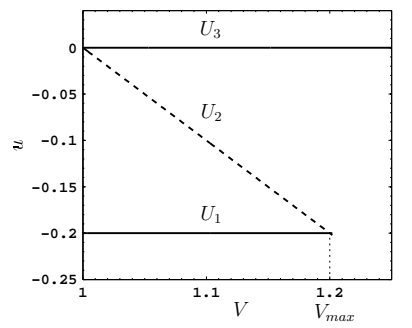

(c)

FIG. 3. Bifurcation diagrams of the single block model. Stationary state, $u$, as a function of the stationary sliding velocity, $V$, for (a) the regularized generalized Coulomb friction force $F_{\varepsilon}\left(a=1, \alpha=0.2, \varepsilon=10^{-4}\right)$, (b) the cubic friction force $F_{c}$, and (c) the piecewise-linear friction force $F_{0}(a=1$, $\alpha=0.2$ ). Solid lines represent stable states (denoted $U_{1}$ and $\left.U_{3}\right)$ and dotted lines are for unstable states $\left(U_{2}\right)$.

\section{BISTABLE SINGLE BLOCK DYNAMICS}

For a single block, (2) reads

$$
\begin{aligned}
\frac{d y}{d t} & =u . \\
m \frac{d u}{d t} & =-F(V+u)+F(V) .
\end{aligned}
$$

The $y$-nullcline is defined by $u=0$, whereas the $u$ nullcline is obtained by solving $F(V+u)=F(V)$ so that the vertical axis $u=0$ always defines in the $(u, y)$ plane the set of fixed points for an isolated block. It is easy to check that the two associated eigenvalues are given by $\lambda_{1}=\frac{-F^{\prime}(V)}{m}, \lambda_{2}=0$ so that the equilibrium straight line is stable (but not asymptotically stable). In (4), the dynamics of the velocity $u$ does not depend on the position $y$ so that system (4) behaves like a one-dimensional dynamical system whose bifurcation diagram is shown in Fig. 3 for the three friction laws (3), where $V$ is taken as the bifurcation parameter. For $V \in\left(a, V_{\max }\right)$, where $V_{\max }$ is the velocity value such that $F\left(V_{\max }\right)$ equals the local maximum in $F$ and $V_{\max }>a$, there exist three fixed points $U_{1}<U_{2}<U_{3}=0$ whose stability is governed by the eigenvalue $\mu_{i}=\frac{-F^{\prime}\left(V+U_{i}\right)}{m}, i \in\{1,2,3\}$, respectively. A saddle-node bifurcation occurs at $V=V_{\max }$ and a transcritical bifurcation takes place at $V=a$. For $V \in\left(a, V_{\max }\right)$, the two fixed points $U_{1}$ and $U_{3}$ are 
stable whereas $U_{2}$ is unstable and behaves like an excitation threshold. For an initial condition below $U_{2}$ the trajectory of the system tends towards $U_{3}=0$, whereas for a sufficiently strong perturbation the system reaches asymptotically the state $U_{1}$, illustrating the excitable dynamics of an isolated block. Depending on the initial state, the system can switch from a neighborhood of $U_{3}$ to $U_{1}$ and vice versa. For the cubic friction force $F_{c}(v)$, the threshold is given by

$$
U_{2}=-\frac{3}{2} V+\frac{9}{8}+\frac{1}{8} \Delta(V)
$$

where $\Delta(V)=\left(-48 V^{2}+72 V-15\right)^{1 / 2}$ (one has $\Delta(V) \epsilon$ $\mathbb{R}$ for $V \in[1 / 4,5 / 4])$. We have

$$
U_{1}=-\frac{3}{2} V+\frac{9}{8}-\frac{1}{8} \Delta(V),
$$

and $V_{\max }=5 / 4$. For the friction force $F_{0}(v)$, the threshold is simply defined as

$$
U_{2}=a-V
$$

the stable fixed point $u_{1}$ is given by

$$
U_{1}=-\alpha a,
$$

and we have $V_{\max }=a(1+\alpha)$. For the regularized generalized Coulomb law $F_{\varepsilon}$, as $\varepsilon \rightarrow 0$ the threshold converges to

$$
U_{2}=(a-V)\left[1+\frac{2}{\alpha}\right]
$$

and the stable fixed point $u_{1}$ to

$$
U_{1}=-V \text {, }
$$

and we have $V_{\max }=a\left(1+\frac{\alpha}{2}\right)$. In the following we are interested in the excitability regime where the velocity of the single block has two stable steady states and we fix a $V$ value in the interval delimited by the two bifurcation points, i.e. $V \in] a, V_{\max }[$. As we will show in the sequel, the bistability property is a key feature for the existence of traveling fronts in the block-spring chain.

\section{TRAVELING WAVES}

Let us consider the block-spring slider model with the regularized generalized Coulomb law $F_{\varepsilon}$. We choose parameters so that each block exhibits a bistable behavior. The parameters of the friction law are those of Fig. 3(a). We initialize the network by applying a perturbation of sufficiently large amplitude to the steady state $U_{3}=0$, and $\left(y_{n}(0)\right)$ is chosen to be constant. A localized perturbation is applied on the first block at the left edge of the network; see Fig. 4 for more details. We consider a finite chain of blocks with free boundary conditions. For the numerical simulations, we use the adaptive Lsoda

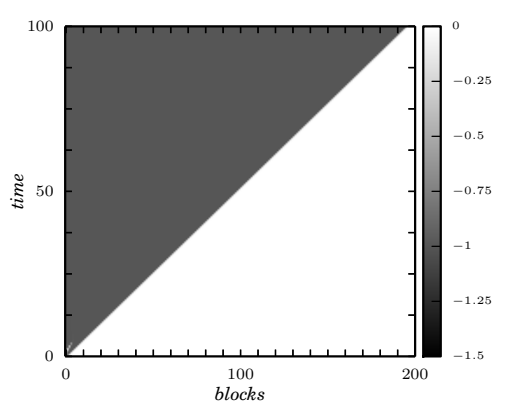

(a)

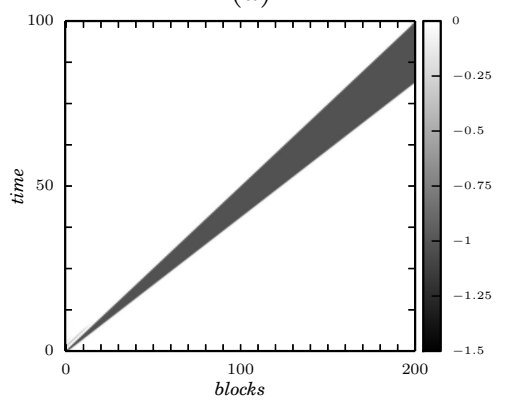

(b)

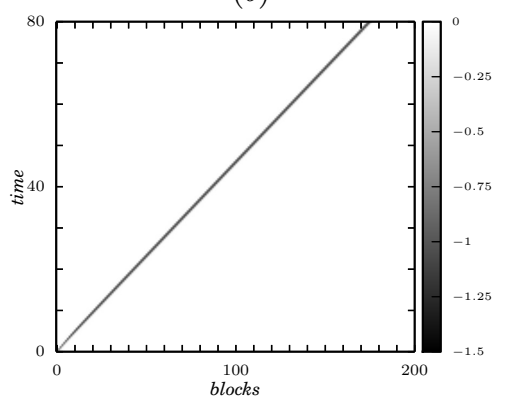

(c)

FIG. 4. Numerical simulations of Eq. (8) with the regularized Coulomb friction force $F_{\varepsilon}$ with the same parameters as in Fig. 3. We display spatiotemporal plots of the velocity variable $u_{n}$ of (a) a traveling front $(k=0.5$ and $V=1.01)$, (b) a broadening pulse ( $k=1$ and $V=1.025)$, and (c) a steadily propagating pulse solution $(k=1$ and $V=1.046)$. An initial perturbation $u_{0}(0)=-10$ is applied on the first block of the chain. Computations are performed for $m=0.15$.

solver and, unless stated otherwise, we take $m=0.15$. We observe the existence of traveling fronts as shown in Fig. 4(a). In addition, two types of pulse solutions are observed: (i) pulse waves with expanding width and (ii) pulse waves with constant shape as plotted in Fig. 4(b) and (c), respectively. Propagating fronts [similar to the one shown in Fig. 4(a)] are the dominant pattern when the threshold is close to the resting state, i.e., for $V$ close to $a\left(\left|U_{2}\right| \ll 1\right)$. The speed of the propagating front increases with the coupling value $k$ but, at the same time, the parameter range where front waves exist shrinks (without vanishing). As the stationary sliding velocity increases, a front to pulse transition occurs 
where the excitation spreads over the network and leads to pulses with expanding width [see Fig. 4(b)]. The rate of expansion of the enlarging pulse decreases as the sliding velocity increases, leading to the existence of a pulse with constant width as shown in Fig. 4(c). For $V \rightarrow V_{\max }$, the threshold approaches the fixed point $U_{1}$ and a perturbation fails to produce a traveling pattern. Qualitatively similar results are obtained for the cubic friction force $F_{c}$ and for the piecewise-linear friction force $F_{0}$.

The profiles of the traveling waves observed in Fig. 4(a,c) are shown in Fig. 5(a,b), respectively, and are compared with those obtained with the cubic law [Fig. 5(c) and 5(d)] and the piecewise-linear law (Fig. 5(e) and 5(f)). The traveling patterns for the three friction forces have similar shapes and mainly contrast in their amplitude, which is determined by the distance between the two stable fixed points. A non-monotonic wave profile is observed for the traveling fronts with the existence of a dip behind the front [see Fig.5(c) and 5(e) whereas the dip is too small to be seen in Fig.5(a)]. The existence of this dip can be justified in the limit of small coupling (see section VI and Appendix B). Interestingly, similar profiles were obtained for traveling fronts in a chain of bistable oscillators [24]. To investigate the influence of inertia on propagation, we show in Fig. 6 the stationary profiles of traveling fronts and traveling pulses for two different values of the mass parameter. It can be seen that fronts and pulses both exist in the over- and underdamped regimes. The main difference between the two regimes occurs during a transitory time, where the underdamped regime leads to transient oscillations at the rear of the fronts (data not shown). The width of the stationary pulse is determined by the spatial extent of the initial perturbation [compare Fig. 5(f), where a single block is excited, with Fig. 7(a), where a broader excitation is used]. The enlarging pulse observed in Figs. 4(b) and 7(b) may be seen as the superposition of two traveling fronts with two different propagation speeds. The initial front is qualitatively similar to the waveform shown in Fig. 5(a) and is followed by a traveling front that propagates in the same direction but with a lower speed and that connects the two stable states in reverse order. The localized pulse waves shown in Figs. 5(b), 5(d), and 5(f) are thus expected to appear when the two traveling fronts have the same speed. These observations are analytically explained in the next section for the piecewise-linear law $F_{0}$.

To check the robustness and the attractivity of the traveling patterns previously observed, we simulate a network where a group of blocks are randomly perturbed. More precisely, we consider a chain of $n=200$ blocks where 20 blocks in the middle of the chain have initial conditions that are uniformly distributed in a fixed interval. Simulations are done for different interval magnitudes and different stationary sliding velocities $V$. Numerical results suggest that the generation of kink-antikink pairs is frequent as shown in Fig. 8. In addition, some initial con-
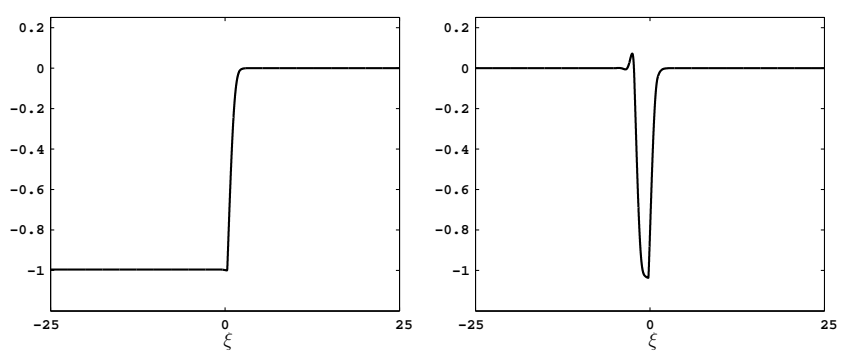

(a)

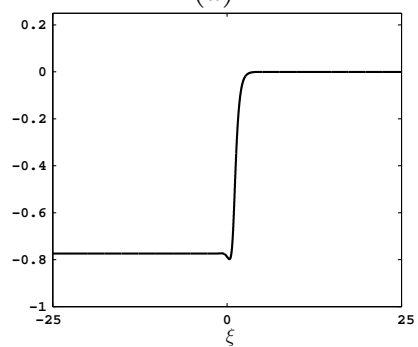

(b)

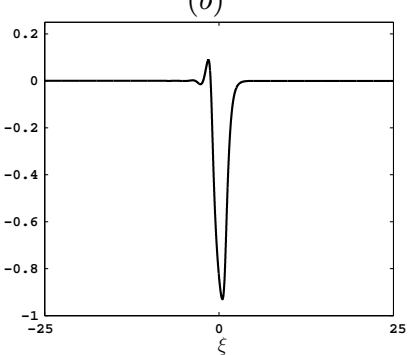

(c)

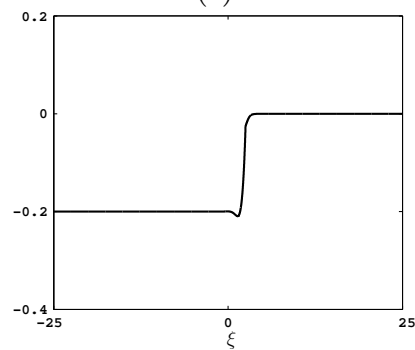

(d)

(e)

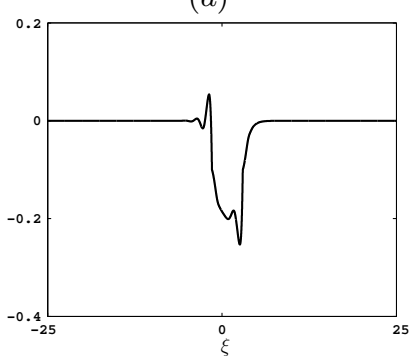

$(f)$

FIG. 5. Plots of the velocity waveforms $u_{n}(t)$ of the blockspring model in the traveling wave coordinate $\xi=n-c t$. The wave profiles in $(a, b)$ are obtained with the regularized generalized Coulomb law $F_{\varepsilon}$ and correspond to the traveling waves shown in Figs. 4(a) and 4(c), respectively. Plots (c) and (d) represent the wave profiles obtained with the cubic friction force, $F_{c}$. Plots (e) and (f) represent the wave profiles obtained with the piecewise-linear friction law $F_{0}$. The wave speed is (a) $c=1.95$, (b) $c=2.21$, (c) $c=3.06$, (d) $c=3.16$, (e) $c=3.16$, and (f) $c=1.45$. For the piecewise-linear law, we use $a=1$ and $\alpha=0.2$. Other parameters are those of Fig. 4 for (a,b), and we take (c) $V=1.025, k=1$ (d) $V=1.18$, $k=2$, (e), $V=1.025, k=1$, and (f) $V=1.1, k=1$.

ditions lead to more complex traveling patterns including pulse trains [see Fig. 8(b)]. An exhaustive numerical investigation will be necessary to refine this statement, but that is beyond the scope of this paper.

\section{CONSTRUCTION OF TRAVELING FRONTS FOR THE PIECEWISE-LINEAR FRICTION FORCE}

Model (2) can be rewritten in terms of velocity as

$$
m \frac{d^{2} u_{n}}{d t^{2}}=k \Delta_{d} u_{n}-\frac{d u_{n}}{d t} F^{\prime}\left(V+u_{n}\right) .
$$



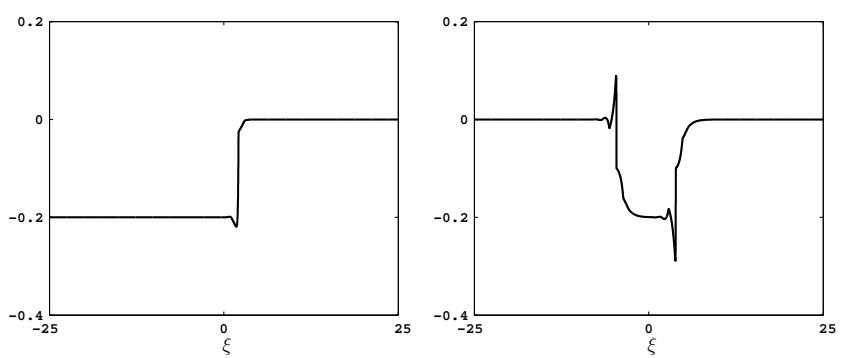

(a)

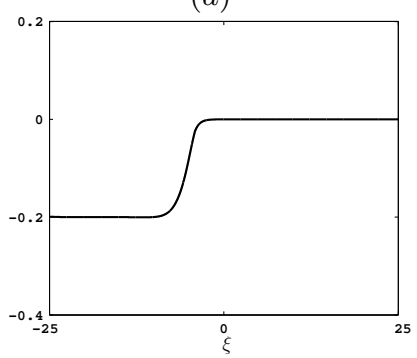

(c) (b)

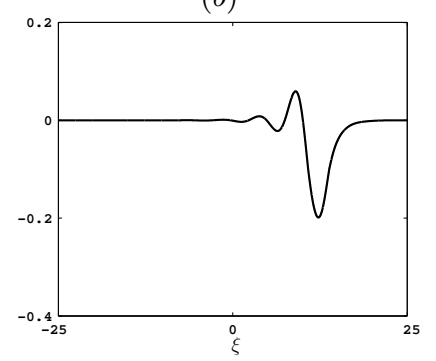

(d)

FIG. 6. Plots of the velocity waveforms $u_{n}(t)$ of the blockspring model in the traveling wave coordinate $\xi=n-c t$. The wave profiles are obtained using the piecewise-linear friction law $F_{0}$ (where $\alpha=0.2$ and $a=1$ ). The stiffness of the spring is fixed to $k=1$. We consider two different values of the mass parameter: (a,b) $m=0.01$ (over-damped regime) and (c,d) $m=100$ (under-damped regime). The stationary sliding velocity is $V=1.025$ for the two traveling fronts shown in (a,c) and $V=1.1$ for the two traveling pulses shown in $(\mathrm{b}, \mathrm{d})$.

A traveling front solution of (8) takes the form

$$
u_{n}(t)=\varphi(n-c t)
$$

where

$$
\varphi(\infty)=U_{3}=0 \quad \text { and } \quad \varphi(-\infty)=U_{1}
$$

with $U_{1} \neq 0$ a stable equilibrium. The function $\varphi$ describes the waveform, and $c$ is the wave speed that has to be determined. Substitution of (9) into (8) gives the advance-delay differential equation

$c^{2} m \varphi^{\prime \prime}(\xi)=k[\varphi(\xi+1)+\varphi(\xi-1)-2 \varphi(\xi)]+c \frac{d}{d \xi} F_{0}[V+\varphi(\xi)]$

where $\xi=n-c t \in \mathbb{R}$ is the travelling wave coordinate. Front solutions connect two different stable steady states as $n \rightarrow \pm \infty$. In contrast, travelling pulses tend towards the same stable equilibrium as $n \rightarrow \pm \infty$.

We consider here the piecewise linear force $F_{0}$, and we assume that each block is in a bistable regime, i.e. we have $V \in(a, a(1+\alpha))$ and $U_{1}=-\alpha a$ as in (7). We assume that the traveling front solution crosses the threshold (6) for only one value of $\xi$. Translation invariance of traveling waves allows us to fix this value to $\xi=0$ and we seek

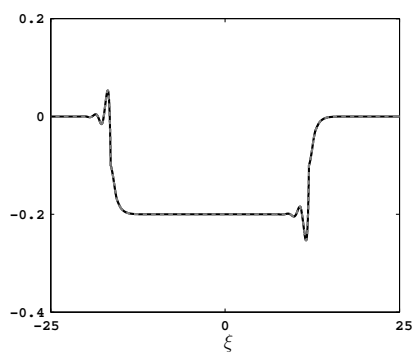

(a)

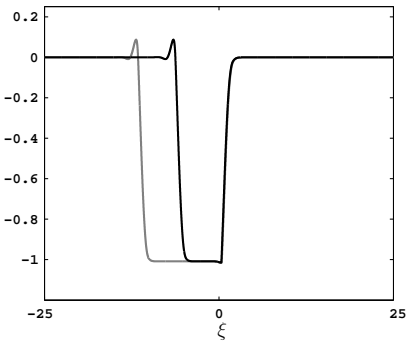

(b)
FIG. 7. Plots of velocity waveforms $u_{n}(t)$ for the block-spring model. (a) Broad stationary pulse formed after the perturbation of a set of blocks ( 15 blocks). Parameters are those of Fig. 5(f). (b) Expanding pulse corresponding to a snapshot of the traveling pattern shown in Fig. 4(b) at two different locations $(n=25, n=50)$. The initial front propagates at a speed $c=2.45$ and the rear front at $c=2$ (the moving frame coordinate is $\xi=n-2 t$ ).

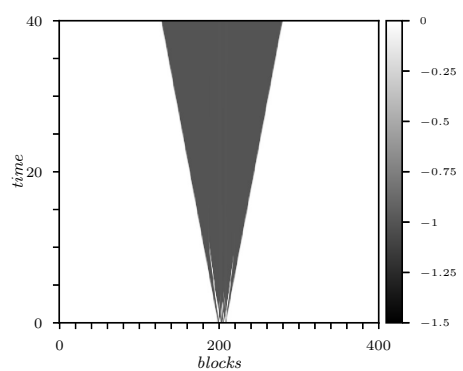

$(a)$

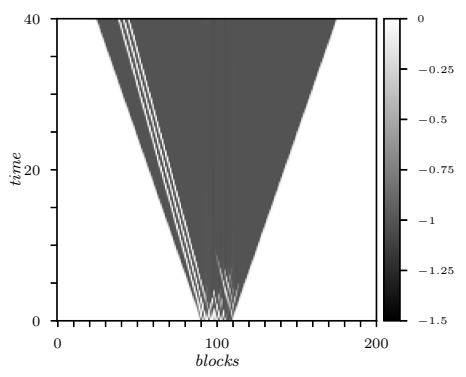

(b)
FIG. 8. Numerical simulations of Eq. (2) with the regularized Coulomb friction force $F_{\varepsilon}$ for random initial conditions. We display two typical spatiotemporal plots of the velocity variable $u_{n}$ for (a) $V=1.02$ and (b) $V=1.03$. A group of 20 blocks in the middle of the chain are perturbed from their equilibrium values. Perturbation is done using random initial conditions where $u_{n}(0)$ is uniformly chosen in the intervals (a) $[-1.23,0.77]$ and (b) $[-1.34,0.66]$.

a solution such that

$$
\left\{\begin{array}{l}
\varphi(\xi)<a-V \text { for } \xi<0 \\
\varphi(0)=a-V \\
\varphi(\xi)>a-V \text { for } \xi>0
\end{array}\right.
$$

Using (12) to simplify the nonlinear term $F_{0}(V+\varphi)$, system (11) takes the form

$$
\begin{aligned}
c^{2} m \varphi^{\prime \prime}(\xi)= & k[\varphi(\xi+1)+\varphi(\xi-1)-2 \varphi(\xi)] \\
& +\frac{c}{a} \varphi^{\prime}(\xi)-\alpha c \delta(\xi),
\end{aligned}
$$

where $\delta(\xi)$ is the Dirac delta function.

Equation (13) is a linear non-autonomous differential equation so that one may attempt to use the Fourier transform to derive an analytic solution. However a certain amount of care is needed to correctly handle the 
Fourier transform of $\varphi$ due to the nonzero boundary condition at $-\infty$. We look for $\varphi(\xi)$ in the form

$$
\left\{\begin{array}{l}
\varphi(\xi)=\alpha a[\psi(\xi)+H(\xi)-1], \\
\psi(\xi) \in L^{2}(\mathbb{R}), \quad \lim _{\xi \rightarrow \pm \infty} \psi(\xi)=0,
\end{array}\right.
$$

where $\psi(\xi)$ has to be determined. Equation (13) is reexpressed in terms of $\psi(\xi)$ and Fourier transform is applied to determine $\psi(\xi)$, and subsequently $\varphi(\xi)$.

Integrating (13), gives

$$
c^{2} m \varphi^{\prime}=k \wedge^{\prime} * \varphi+\frac{c}{a} \varphi+\alpha c(1-H),
$$

where $\wedge(\xi)=\max (1-|\xi|, 0)$ is the tent function, and where we used for any $f \in L_{\text {loc }}^{1}(\mathbb{R})$,

$$
\left(\wedge^{\prime} * f\right)(\xi)=\int_{\xi}^{\xi+1} f(s) d s-\int_{\xi-1}^{\xi} f(s) d s .
$$

Note that (15) together with (10) remains equivalent to the original problem (13)-(10). Injecting (14) into (15), gives

$$
c^{2} m \psi^{\prime}-\frac{c}{a} \psi-k \wedge^{\prime} * \psi=k \wedge-c^{2} m \delta,
$$

where we used the property $\wedge^{\prime} *(H-1)=\wedge$. Taking the Fourier transform as $\widehat{\psi}(\lambda)=\int_{\mathbb{R}} e^{-2 \pi i \lambda \xi} \psi(\xi) d \xi$ in (17), we obtain

$$
\left[2 i \pi \lambda c^{2} m-\frac{c}{a}-k 2 i \pi \lambda \operatorname{sinc}^{2}(\lambda)\right] \widehat{\psi}(\lambda)=k \operatorname{sinc}^{2}(\lambda)-c^{2} m,
$$

where we used $\widehat{\wedge}(\lambda)=\operatorname{sinc}^{2}(\lambda)$ with $\operatorname{sinc}(\lambda)=$ $\sin (\pi \lambda) / \pi \lambda$. Let us introduce

$$
\widehat{K}(\lambda)=\left(2 i \pi \lambda\left[c^{2} m-k \operatorname{sinc}^{2}(\lambda)\right]-\frac{c}{a}\right)^{-1}
$$

where one has $\widehat{K}(\lambda), K(\xi) \in L^{2}(\mathbb{R})$ ( $K$ denotes the inverse Fourier transform of $\hat{K})$. From $\frac{d \widehat{K}}{d \lambda} \in L^{1}(\mathbb{R})$ and using $-2 i \pi \xi K=\mathcal{F}^{-1}\left(\frac{d \widehat{K}}{d \lambda}\right) \in L^{\infty}(\mathbb{R})$, one has $\lim _{\xi \rightarrow \pm \infty} K(\xi)=0\left(\mathcal{F}^{-1}\right.$ denotes the inverse Fourier transform). From (18), we obtain

$$
\psi=k K * \wedge-c^{2} m K .
$$

Since $\wedge \in L^{1}(\mathbb{R})$ we have $K * \wedge \in L^{2}(\mathbb{R})$, and because $K, \wedge \in L^{2}(\mathbb{R})$ then $K * \wedge \in C^{0}(\mathbb{R})$ decays to zero when $\xi \rightarrow \pm \infty$. Consequently, $\psi(\xi)$ given by (19) satisfies the properties assumed in (14), and it defines a unique solution in $L^{2}(\mathbb{R})$. Therefore (14) is a solution of (13) with boundary conditions (10). Regularity properties of $\varphi(\xi)$ can be inferred from the following identity obtained from (14) and (17)

$$
\frac{c^{2} m}{\alpha a} \varphi^{\prime}=\frac{c}{a} \psi+k \wedge^{\prime} * \psi+k \wedge .
$$

This implies $\varphi^{\prime} \in L_{l o c}^{1}(\mathbb{R})$ ( since $\wedge^{\prime} * \psi \in L^{2}(\mathbb{R})$ ) and thus ones has $\varphi \in C^{0}(\mathbb{R})$. We also get from (15) that $\varphi^{\prime} \in C^{0}\left(\mathbb{R}^{+}\right) \cap C^{0}\left(\mathbb{R}^{-}\right)$, hence $\varphi \in C^{1}\left(\mathbb{R}^{+}\right) \cap C^{1}\left(\mathbb{R}^{-}\right)$, and thus (15) gives $\varphi^{\prime} \in C^{1}\left(\mathbb{R}^{+}\right) \cap C^{1}\left(\mathbb{R}^{-}\right)$. We get finally

$$
\varphi \in C^{2}\left(\mathbb{R}^{+}\right) \cap C^{2}\left(\mathbb{R}^{-}\right) \cap C^{0}(\mathbb{R})
$$

From the analytical expression of $\varphi$, we can derive an equation to determine the wave speed of the front. Using (14), we get

$$
\frac{\varphi(\xi)+\varphi(-\xi)}{2}=\frac{\alpha a}{2}[\psi(\xi)+\psi(-\xi)-1]
$$

where $\psi$ is defined by (19) (note that we used $H(-\xi)=$ $1-H(\xi)$ to eliminate the Heaviside function). Using the threshold condition $\varphi(0)=a-V$ from (12) together with (21) and (19), we obtain that the wave speed satisfies

$$
\alpha a\left[\psi\left(0^{+}\right)+\psi\left(0^{-}\right)-1\right]+2(V-a)=0 .
$$

This scalar equation allows us to compute $c$ numerically using a Newton-type method. Computation of $K$ is done using a Gauss-Konrod quadrature formula in a truncated interval $\left[-10^{6}, 10^{6}\right]$ (an alternate approach would be to use the residue theorem with a numerical computation of the poles of $K[25]$ ). We restrict to $c>0$ (the case $c<0$ can be deduced by symmetry, see section VII). A plot of the resulting analytical profile (14) is shown in Fig. 9(a) and compared with the numerical simulation of (8). A perfect matching is realized between the two trajectories. The typical dependence of the wave speed on the stationary sliding velocity, $V$, and on the coupling, $k$, is shown in Fig. 9(b).

\section{ANTI-CONTINUUM LIMIT}

In this section, the small coupling limit is explored. We consider the case $c>0$ (see Sec. VII for the case $c<0$ ). From (17) and (19) with $k \rightarrow 0$, we have the leading order equation

$$
c^{2} m K^{\prime}-\frac{c}{a} K-k \wedge^{\prime} * K=\delta
$$

where we look for a solution of the form

$$
K=K_{0}+k K_{1}+\mathcal{O}\left(k^{2}\right) .
$$

Inserting (24) in (23), and equating orders of leading terms in $k$, we obtain

$$
\begin{aligned}
& c^{2} m\left(K_{0}^{\prime}-\nu K_{0}\right)=\delta, \\
& c^{2} m\left(K_{1}^{\prime}-\nu K_{1}\right)=\wedge^{\prime} * K_{0},
\end{aligned}
$$

where $\nu^{-1}=$ cam. Observe that (25) has the unique bounded solution

$$
K_{0}(\xi)=-\frac{1}{c^{2} m} e^{\nu \xi} H(-\xi),
$$




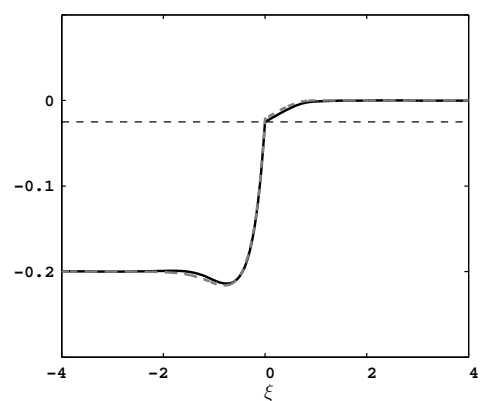

(a)

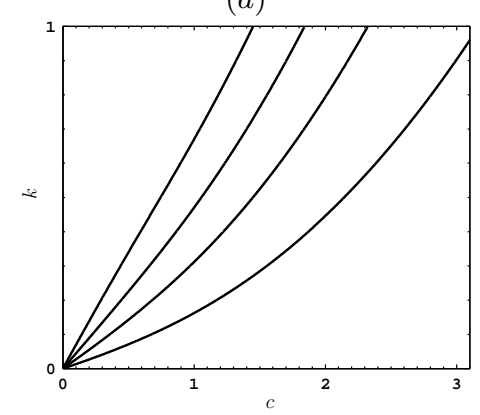

(b)

FIG. 9. (a) Travelling front solution $\varphi(\xi)$ computed from the explicit formula (14) where $k=0.3, V=1.025, a=1$, and $\alpha=0.2$ (full line). The trajectory is indistinguishable from the one obtained from the numerical simulation of the chain. The asymptotic approximation (29) obtained for $k \ll 1$ is also shown (dashed gray line). We obtain $c=1.55$ from the threshold condition (12) (the dashed line defines the threshold $\left.u_{2}=a-V\right)$. (b) Wave speed curves in the $(c, k)$ plane obtained from (22) for $V=1.025,1.05,1.075$, and 1.1 (from right to left, respectively).

where $K_{0} \in L^{1}(\mathbb{R})$, hence the solution of (26) reads

$$
\begin{aligned}
K_{1} & =K_{0} * \wedge^{\prime} * K_{0}=\wedge * K_{0} * K_{0}^{\prime}, \\
& =\frac{1}{c^{2} m} \wedge * K_{0}+\nu \wedge * K_{0} * K_{0},
\end{aligned}
$$

where we used $K_{0}^{\prime}=\frac{1}{c^{2} m} \delta+\nu K_{0}$. Using (19) with (27) and (28), the approximation for $\varphi$ up to $\mathcal{O}\left(k^{2}\right)$ reads

$$
\begin{aligned}
\varphi(\xi)= & \alpha a\left(e^{\nu \xi}-1\right) H(-\xi)+\alpha a k\left[-c^{2} m K_{1}(\xi)\right. \\
& \left.+\left(K_{0} * \wedge\right)(\xi)\right]+\mathcal{O}\left(k^{2}\right),
\end{aligned}
$$

where we used the identity $H(-\xi)=1-H(\xi)$.

Expression (29) allows to obtain an approximation of the wave speed $c$ for small $k$. From $\varphi(0)=a-V$ and (29), we get

$$
\begin{aligned}
a-V & =\alpha a k\left(-c^{2} m K_{1}(0)+\left(K_{0} * \wedge\right)(0)\right)+\mathcal{O}\left(k^{2}\right), \\
& =-\alpha k c\left(\wedge * K_{0} * K_{0}\right)(0)+\mathcal{O}\left(k^{2}\right), \\
& :=S(c) k+\mathcal{O}\left(k^{2}\right) .
\end{aligned}
$$

We obtain after some calculations (see Appendix A)

$$
S(c)=2 \alpha m a^{3}-\frac{\alpha a^{2}}{c}\left((2 a m c+1) e^{-1 / a m c}+1\right) .
$$

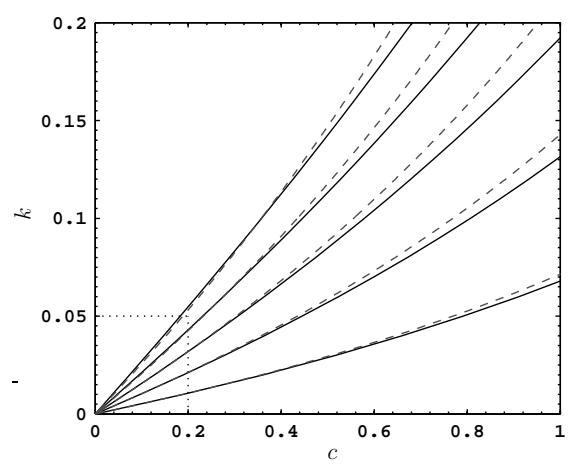

(a)

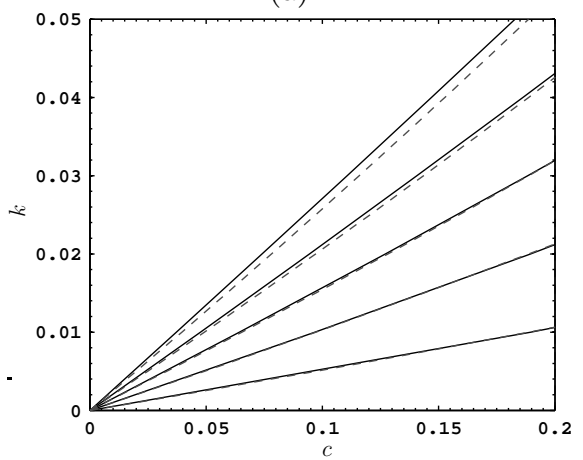

(b)

FIG. 10. (a) Speed curves of the traveling front solution in the $(c, k)$ plane for $V=1.0025,1.005,1.0075$ and 1.01 (from right to left, respectively). Curves $(c, k)$ computed with (34) (dashed gray line) accurately describe the exact curves $(c, k)$ computed with (22) (black continuous) in the limit $c \rightarrow 0$. (b) A zoom of the dashed square region in panel (a) is shown. Parameter values are $\alpha=0.2$ and $a=1$.

To approximate $c$, we drop $\mathcal{O}\left(k^{2}\right)$ terms in (30). The wave speed can be estimated from the solution of

$$
\nu-2+(\nu+2) e^{-\nu}=\frac{V-a}{\alpha m a^{3} k}
$$

where $\nu^{-1}=a c m$. It can be shown that the left-hand side of (32) defines a bijective function on $\mathbb{R}$ that passes through the origin so that (32) admits a unique solution. Let us fix the values of $V$ and $a$, and look for solutions $c \approx 0$ when $k \approx 0$. Observing the exponential decay $e^{-\nu} \rightarrow 0$ as $c \rightarrow 0$, we have, from (31) and (30) the leading order approximation

$$
\nu=2+\frac{V-a}{\alpha m a^{3} k}
$$

for $k, c \rightarrow 0$. Therefore we obtain the following approximation for the wave speed:

$$
c \sim \frac{1}{2 a m+\frac{V-a}{\alpha a^{2} k},}
$$

where the leading-order approximation reads $c \sim \frac{\alpha a^{2} k}{V-a}$. Formula (34) was derived under the assumption that $c$ 
is small for $k$ small, and one can easily check that $c$ given by (34) satisfies $c \rightarrow 0$ as $k \rightarrow 0$. To evaluate the accuracy of the asymptotic approximation (34), we compare in Fig. 10 the $(c, k)$ curves obtained from $(22)$ with those computed from (34) for different sliding velocities $V$. The asymptotic approximation (29) of the waveform is compared with the exact solution [see Fig. 9(a)]. A good matching between the two wave profiles is found. Monotonicity analysis of the approximated waveform (29) shows that the velocity profile is nonmonotonic, i.e., a dip always exists behind the front (see Appendix B).

\section{REVERSE TRAVELING FRONTS AND PULSES}

In the previous section, we constructed traveling fronts connecting the two stable equilibria $U_{1}=-\alpha a$ (when $n \rightarrow-\infty$ ) and 0 (when $n \rightarrow+\infty$ ). In this analysis we have restricted our attention to traveling fronts with positive velocity $c(V)$ (for now we consider the dependency of front velocity in $V$ and discard the other parameters). Using symmetry arguments, we show in the sequel the existence of travelling fronts with negative velocity satisfying the same boundary conditions. We also deduce the existence of traveling fronts with positive velocity satisfying reverse boundary conditions $\left(u_{n} \rightarrow-\alpha a\right.$ when $n \rightarrow+\infty$ and $u_{n} \rightarrow 0$ when $\left.n \rightarrow+\infty\right)$.

Let us start with some symmetry considerations. Consider the advance-delay equation (11) with boundary conditions

$$
\varphi(-\infty)=U_{1}, \quad \varphi(+\infty)=U_{3} .
$$

This problem admits the invariance

$$
\varphi(\xi) \rightarrow \varphi(-\xi), \quad c \rightarrow-c, \quad\left(U_{1}, U_{3}\right) \rightarrow\left(U_{3}, U_{1}\right) .
$$

Moreover, the piecewise-linear friction force $F_{0}$ is antisymmetric about $v=a$, i.e., we have

$$
F_{0}(a+h)+F_{0}(a-h)=2-\alpha, \quad \text { for all } h \in \mathbb{R} .
$$

As a consequence, one can readily check that (11) with (35) are invariant by the one-parameter family of transformations

$$
\begin{array}{r}
\varphi \rightarrow-\lambda-\varphi, \quad V \rightarrow 2 a+\lambda-V, \\
\left(U_{1}, U_{3}\right) \rightarrow\left(-\lambda-U_{1},-\lambda-U_{3}\right),
\end{array}
$$

where $\lambda \in \mathbb{R}$ is arbitrary.

Now let us use the above invariances in order to obtain reverse traveling fronts. We define $\tilde{\zeta}=-\alpha a-\varphi$, so that $\tilde{\zeta}$ and $\varphi$ connect stable equilibria in reverse order at infinity. Applying invariance (37) for $U_{3}=0$ and $\lambda=\alpha a=-U_{1}$, it follows that $\varphi$ is a solution of (11) if and only if $\tilde{\zeta}$ is a solution of the same equation with modified sliding velocity $\tilde{V}=a(2+\alpha)-V$. From the results of Sec. V, this problem admits for all $\tilde{V} \in(a, a(1+\alpha))$ a front solution $\tilde{\zeta}$ satisfying the boundary conditions $\tilde{\zeta}(-\infty)=-\alpha a, \tilde{\zeta}(+\infty)=0$, with velocity $c(\tilde{V})>0$. From invariance $(36)$, this equation possesses another front solution $\zeta(\xi)=\tilde{\zeta}(-\xi)$ with velocity $-c(\tilde{V})<0$, which satisfies the boundary conditions $\zeta(+\infty)=-\alpha a, \zeta(-\infty)=0$. It follows that for all $V \in(a, a(1+\alpha))$, Eq. (11) with sliding velocity $V$ admits the front solution $\tilde{\varphi}=-\alpha a-\zeta$, satisfying the boundary conditions (10) and having a negative velocity $-c(a(2+\alpha)-V)$. Consequently, the search of front solutions of (10) and (11) can be reduced to the case $c>0$ examined in Sec. V, since all fronts with $c<0$ can be deduced by symmetry.

Furthermore, $\varphi(\xi)=\tilde{\varphi}(-\xi)=-\alpha a-\tilde{\zeta}(\xi)$ defines another solution of (11) with sliding velocity $V$. This front has a positive velocity $c(\tilde{V})=c(a(2+\alpha)-V)$ and satisfies the reverse boundary conditions

$$
\varphi(-\infty)=0, \quad \varphi(+\infty)=-\alpha a .
$$

The coexistence of this reverse front and the front satisfying (10) and (11) with the different velocity $c(V)$ can be used to understand the broadening of pulses reported in Sec. IV, as well as the existence of steadily propagating pulses observed for particular sliding velocities. Indeed, we can see from Fig. 10(b) that the function $V \mapsto c(V)$ is decreasing [this is also clear from the leading order approximation (34)]. Consequently, gluing the above two fronts to form a pulse decaying to 0 at infinity, the trailing front (at the rear of the propagating pulse) will be slower if $V<\tilde{V}$, resulting in a broadening of the pulse. This regime occurs for $V \in\left(a, a\left(1+\frac{\alpha}{2}\right)\right)$. In the critical case $V=a\left(1+\frac{\alpha}{2}\right)$, we have $V=\tilde{V}$ and the two fronts have identical velocities, thereby maintaining a steadily propagating pulse [this case is shown in Fig. 5(f)]. Conversely, for $V \in\left(a\left(1+\frac{\alpha}{2}\right), a(1+\alpha)\right)$, the trailing front is faster and no pulse wave can propagate. Starting from an initial bump condition, an annihilation occurs when the trailing front reaches the leading front. In conclusion, the condition for the existence of broadening pulses reads

$$
V<V^{*} \quad \text { where } \quad V^{*}=a\left(\frac{\alpha}{2}+1\right) .
$$

For $V>V^{*}$, pulse fails to propagate whereas for $V=V^{*}$ a stable pulse is observed, with a width determined by the initial perturbation. In the small coupling limit, this pulse has a wave speed $c \sim 2 a k$ according to approximation (34).

\section{DISCUSSION}

We studied localized traveling waves in a nonlinear lattice describing a block-spring chain sliding down a slope and experiencing friction. Wave propagation was illustrated for different spinodal friction laws. For a particular range of stationary sliding velocities, the medium is made of blocks exhibiting bistabilities, and it supports 
nonlinear transition waves (wave fronts). Interesting connections can be made with recent results on waves in bistable lattices $[5,26]$. For an idealized piecewise-linear friction force, we constructed analytically traveling fronts and analyzed their wave speeds. In contrast with the discrete Nagumo equation, propagating fronts exist at small coupling values, i.e., propagation failure does not occur at weak-coupling strengths. As already observed in a different context [27], the traveling pulses are shaped by the concatenation of two traveling front solutions, and pulse propagation failure occurs when the back wave is faster than the front wave. We determined analytically the parameter range where pulses of constant width occur, i.e., the leading front and the trailing front have the same velocity. It is worth noting that this analysis does not rely on a time scale separation and differs from the asymptotic construction of pulses done in [28]. In particular, the pulse width is not determined by the equality of the velocity of the two fronts but depends on the initial excitation.

The present study is also of interest for the under-

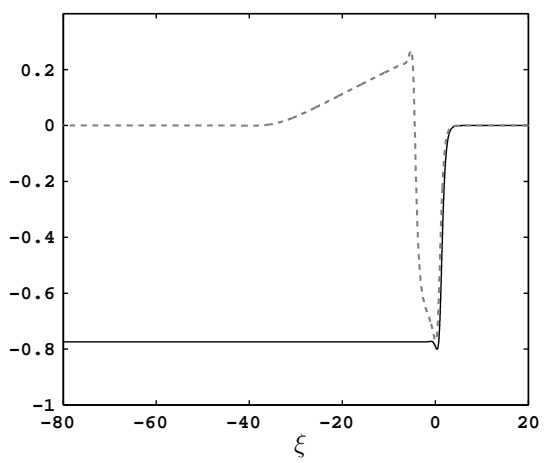

FIG. 11. Comparison of a front solution $u_{n}(t)$ of $(2)$ with velocity $c=c_{f} \approx 0.79$ (solid line) with a pulse (velocity $\left.c=c_{B K} \approx 15.70\right)$ supported by the Burridge-Knopoff model (dotted line). The velocity waveforms are plotted in the traveling-wave coordinate $\xi=n-c t$. Computations are performed for the cubic friction law $F_{c}$ and the following parameters : $\gamma=0.05, k_{c}=10$, and $V=1.025$. The pulse velocity $c_{B K}$ is well approximated by the (rescaled) front velocity $c_{f} / \gamma \approx 15.85$.

standing of the dynamics of the Burridge-Knopoff model, where the time evolution of the system is given by

$$
\gamma \ddot{y}_{n}=k_{c} \Delta_{d} y_{n}-F\left(V+\dot{y}_{n}\right)-y_{n} .
$$

Let us define $y_{n}(t)=-F(V)+\gamma z_{n}(t / \gamma)$ and $k=\gamma k_{c}$. Assuming $\gamma \ll 1$, then, the Burridge-Knopoff model (40) can be approximated in the fast time scale by

$$
\ddot{z}_{n}=k \Delta_{d} z_{n}-F\left(V+\dot{z}_{n}\right)+F(V)
$$

which coincides with (2). Equation (41) is obtained from (40) neglecting the $\gamma z_{n}$ term, i.e., model (2) does not incorporate a "dynamic recovery" and only describes the initial excitation of the Burridge-Knopoff system. Therefore, for small $\gamma$ values, one may expect that the front waves of (2) provide useful information on the dynamics of pulse propagation in the Burridge-Knopoff model (40). More precisely, fronts approximate the transition region from the ground state to the excited state. This is shown in Fig. 11 where the fast time scale of the BurridgeKnopoff model is accurately reproduced by model (2). In addition, numerical experiments suggest that the wave speed of the solitary wave of the Burridge-Knopoff model is well approximated by the (rescaled) velocity of kinks of (2); see caption of Fig. 11. In the case of the piecewiselinear friction law $F_{0}$, the computation of the front velocity is much simpler than for the solitary wave of the Burridge-Knopoff model. A detailed study of solitary waves in the excitable Burridge-Knopoff model is presented in [7].

Interesting numerical problems left open in this study concern the continuation of stationary front and pulse solutions and the analysis of their linear stability. For the piecewise-linear friction law, front stability will be analytically addressed in a future work (in that case, the discontinuity of the friction force introduces some difficulties).

\section{ACKNOWLEDGMENTS}

We thank the reviewers for their helpful comments on the manuscript. We are grateful to A. Vainchtein, J. P. Françoise, M. Herrmann, H. de Jong, and B. Lombard for helpful comments and stimulating discussions.

\section{Appendix A: Computation of $S(c)$}

We compute here the explicit expression of $S(c)=$ $-\alpha c\left(\wedge * K_{0} * K_{0}\right)(0)$. We reexpress $K_{0}$ as $K_{0}(\xi)=$ $-\frac{G(\xi)}{c^{2} m}$ where $G(\xi)=e^{\nu} H(-\xi)$, hence we have

$$
S(c)=-\frac{\alpha}{c^{3} m^{2}}(\wedge * G * G)(0) .
$$

We have

$$
(G * G)(-s)=\int_{-s}^{0} G(\tau) G(-s-\tau) d \tau=s e^{-\nu s} H(s)
$$

with $s>0$, therefore

$$
\begin{aligned}
(\wedge * G * G)(0) & =\int_{\mathbb{R}} \wedge(\tau)(G * G)(-\tau) d \tau \\
& =\int_{0}^{1}(1-\tau)\left(\tau e^{-\nu \tau}\right) d \tau \\
& =\frac{\nu+e^{-\nu} \nu-2+2 e^{-\nu}}{\nu^{3}} \\
& =\frac{e^{-\nu}}{\nu^{3}}(2+\nu)+\frac{-2+\nu}{\nu^{3}}
\end{aligned}
$$


with $\nu=(\mathrm{cam})^{-1}$. We further calculate

$$
\frac{e^{-\nu}}{\nu^{3}}(2+\nu)+\frac{-2+\nu}{\nu^{3}}=-\nu^{-2}\left[2 \nu^{-1}-\left(2 \nu^{-1}+1\right) e^{-\nu}-1\right]
$$

Inserting (A2) into (A1) gives

$$
S(c)=\frac{\alpha \nu^{-2}}{c^{3} m^{2}}\left[2 \nu^{-1}-1-\left(2 \nu^{-1}+1\right) e^{-\nu}\right],
$$

and (31) follows.

\section{Appendix B: Profile of the approximated front}

From (28) and (29) one has the following asymptotic approximation of the waveform

$$
\varphi(\xi)=\alpha a\left(e^{\nu \xi}-1\right) H(-\xi)-\alpha c k \wedge * K_{0} * K_{0}(\xi)+\mathcal{O}\left(k^{2}\right) .
$$

We note $\varphi_{1}=\wedge * K_{0} * K_{0}$ and we calculate $\varphi_{1}=0$ for $\xi \geq 1$ and

$$
\begin{aligned}
\varphi_{1}(\xi)= & \frac{1}{m^{2} c^{4} \nu^{3}}\left((2+\nu(1-\xi)) e^{\nu(\xi-1)}\right. \\
& \left.+(2-\nu(1+\xi)) e^{\nu(\xi+1)}+(2 \nu \xi-4) e^{\nu \xi}\right)
\end{aligned}
$$

for $\xi \leq-1$. For $\xi \ll 0$ we obtain the following approximation:

$$
\varphi_{1}(\xi) \sim-\frac{2(\cosh (\nu)-1)}{m^{2} c^{4} \nu^{2}} \xi e^{\nu \xi} .
$$

Therefore the traveling front takes the leading form

$$
\varphi(\xi) \sim-\alpha a+\frac{2 \alpha(\cosh (\nu)-1) k}{m^{2} c^{3} \nu^{2}} \xi e^{\nu \xi}
$$

as $\xi \ll 0$. Using $c \sim \frac{\alpha a^{2} k}{V-a}$ we have

$$
\varphi(\xi) \sim-\alpha a+2(V-a)(\cosh (\nu)-1) \xi e^{\nu \xi}
$$

which is a decreasing function of $\xi$ for $\xi \ll 0$. The leading approximation of the wavefront is zero for $\xi \geq 1$ and has a decreasing profile for $\xi$ sufficiently small, therefore the wavefront is nonmonotonic and presents (at least) one dip after the front. Notice that the function occuring on the righthand side of (B1) has a minimum at $\xi=-1 / \nu$ that may be used to approximate the dip location.
[1] Y. S. Kivshar, F. Zhang, and S. Takeno, J. Phys. D 113, 248 (1998).

[2] P. Maniadis and S. Flach, Europhys. Lett. 74, 452 (2006).

[3] J. F. Rhoads, S. W. Shaw, and K. L. Turner, J. Dyn., Meas., Control 132, 034001 (2010).

[4] K. Leung and Z. Néda, Phys. Rev. Lett. 85, 662 (2000).

[5] J. Raney, N. Nadkarni, C. Daraio, D. Kochmann, J. Lewis, and K. Bertoldi, Proc. Natl. Acad. Sci. USA 113, 9722 (2016).

[6] R. Burridge and L. Knopoff, Bull. Seismol. Soc. Am. 57, 341 (1967).

[7] J. Morales, G. James, and A. Tonnelier, Res. Rep. No. RR-8966, INRIA, hal-01411897 (2016).

[8] E. M. Izhikevich, Dynamical systems in neuroscience: The geometry of excitability and bursting (The MIT Press, Cambridge, MA, 2007).

[9] G. Ermentrout and D. Terman, Mathematical Foundations of Neuroscience (Springer-Verlag, New-York, 2010).

[10] J. D. Murray, Mathematical Biology, Biomathematics (Springer-Verlag, Berlin, 1993).

[11] J. H. E. Cartwright, E. Hernández-García, and O. Piro, Phys. Rev. Lett. 79, 527 (1997).

[12] J. H. E. Cartwright, V. M. Eguíluz, E. Hernández-García, and O. Piro, Int J. Bif. Chaos 9, 2197 (1999).

[13] C. B. Muratov, Phys. Rev. E 59, 3847 (1999).

[14] N. Flytzanis, S. Crowley, and V. Celli, J. Phys. Chem. Solids 38, 539 (1977).
[15] J. W. Cahn, J. Mallet-Paret, and E. S. Van Vleck, SIAM J. Appl. Math. 59, 455 (1998).

[16] A. Tonnelier, Phys. Rev. E 67, 036105 (2003).

[17] C. E. Elmer and E. S. Van Vleck, SIAM J. Appl. Math. 65, 1153 (2005).

[18] W. Atkinson and N. Cabrera, Phys. Rev. 138, A763A766 (1965)

[19] L. Truskinovsky and A. Vainchtein, SIAM J. Appl. Math. 66, 533 (2005).

[20] A. Vainchtein, J. Mech. and Phys. Solids 58, 227 (2010).

[21] L. Truskinovsky and A. Vainchtein, Phys. Rev. E 90, 042903 (2014).

[22] P. Rosakis and A. Vainchtein, J. Nonlin. Sci. 23, 1089 (2013).

[23] H. Schwetlick and J. Zimmer, SIAM J. Math. Anal. 41, 1231 (2009).

[24] M. Duanmu, N. Whitaker, P. G. Kevrekidis, A. Vainchtein, and J. Rubin, J. Phys. D 325, 25 (2016).

[25] A. Vainchtein and E. S. Van Vleck, Phys. Rev. B 79, 144123 (2009).

[26] N. Nadkarni, A. Arrieta, C. Chong, D. Kochmann, and C. Daraio, Phys. Rev. Lett. 116, 244501 (2016).

[27] A. Tonnelier, J. Phys. D 210, 118 (2005).

[28] A. Carpio and L. Bonilla, SIAM J. Appl. Math. 63, 619 (2003). 